\title{
DENGUE INFECTION
}

nature DISEASE

REVIEWS PRIMERS

Dengue is a mosquito-borne disease caused by infection with dengue virus (DENV). Clinically, the disease can range from a mild febrile illness (previously called dengue fever) through to dengue with warning signs and severe dengue, which includes what were previously called dengue haemorrhagic fever (DHF) and dengue shock syndrome (DSS).

\section{EPIDEMIOLOGY}

DENVs are transmitted by Aedes aegypti

mosquitoes and are widespread throughout the tropics. The global expansion of DENV s began with the South-East Asian dengue pandemic in the 1950s and was facilitated by the effects of globalization, such as increased air travel. Over half of the world's population are at risk of DENV infection. Each year, an estimated 360 million DENV infections occur, resulting in 2 million cases of severe disease and 21,000 deaths.

\section{PREVENTION}

Prevention focuses on mosquito control using strategies such as larval source reduction,

insecticide spraying and community

outreach and education. Although some

countries, such Singapore and Cuba, achieved

temporary dengue control using

these approaches, their

inconsistent application

and the introduction

of DENVs from

neighbouring

countries

eventually led to

the re-emergence

of epidemic

DENV transmission.

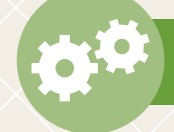

PATHOPHYSIOLOGY

High viral loads and fever

follow $4-7$ days later

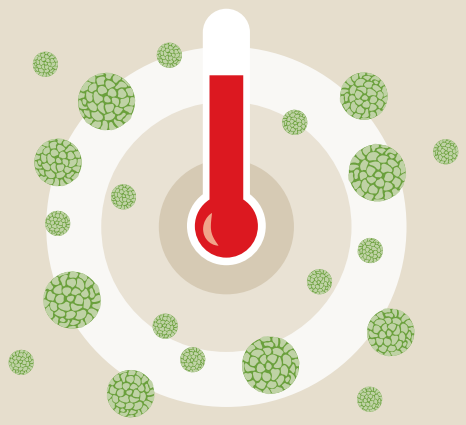

..............

Plasma leakage and abnormal haemostasis underlie DHF and DSS. Although their causes are not well understood, antibody dependent enhancement and the DENV protein NS1 are
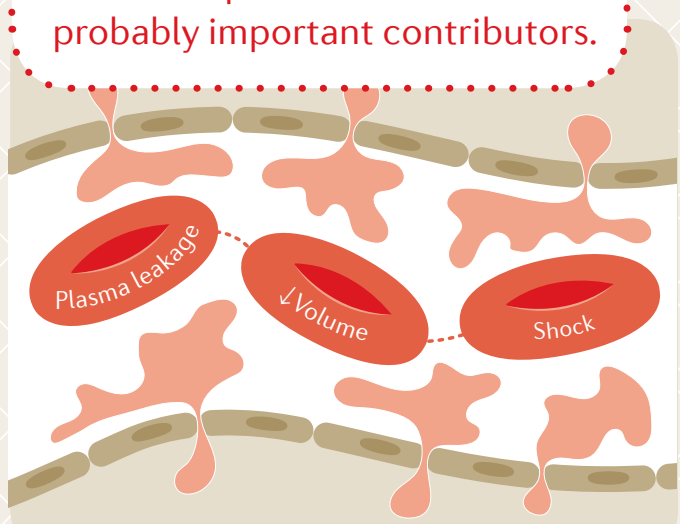

\section{(1) OUTLOOK}

To succeed in preventing dengue, mosquito control efforts will need to be sustained in the long term and conducted at the regional leve to prevent the spread of DENVs from adjacent endemic areas. This effort will be aided by the

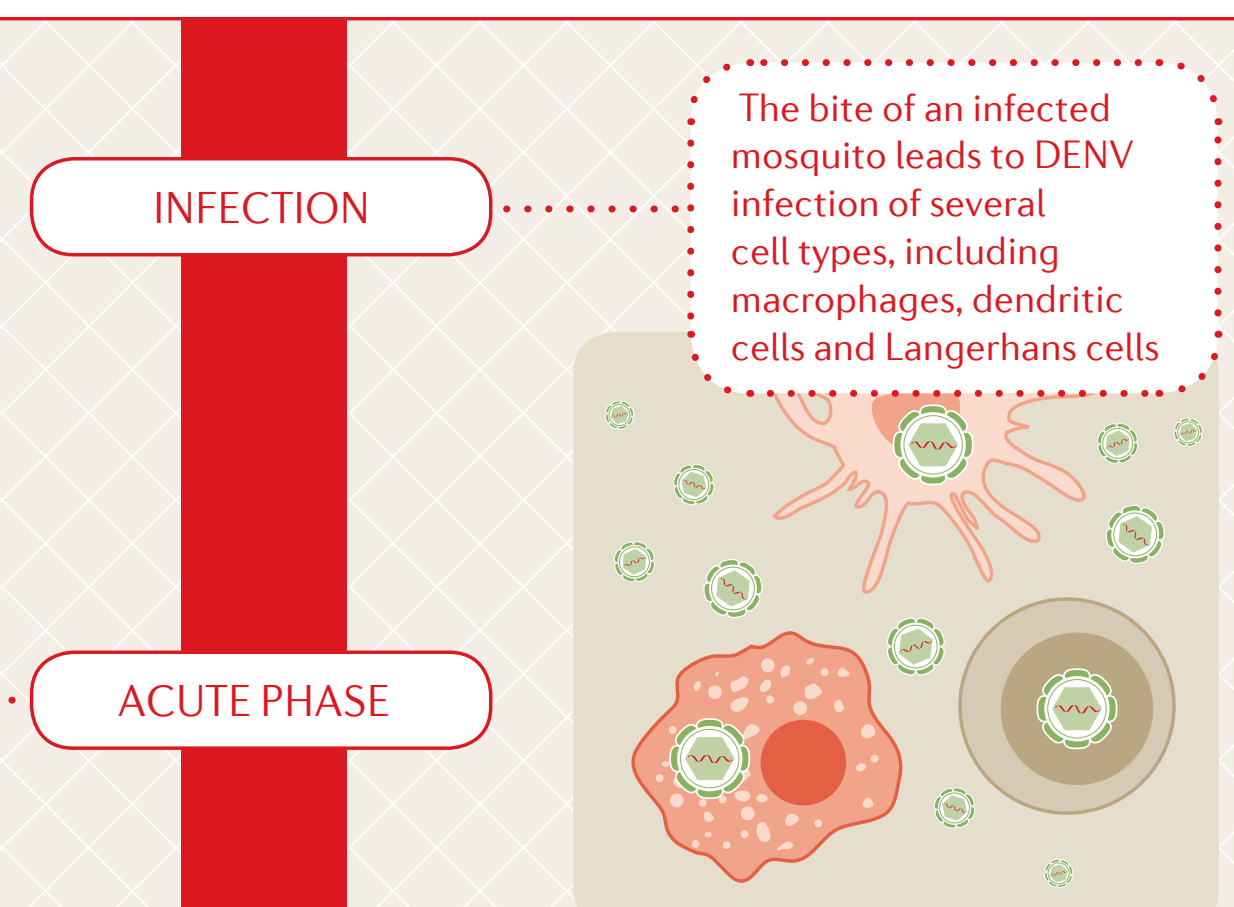

For the Primer, visit doi:10.1038/nrdp.2016.5

\section{DIAGNOSIS}

The 2009 WHO guidelines are used to clinically classify dengue and identify patients at risk of progressing from mild to severe illness. Diagnosis of presumptive dengue with or without warning signs is made if the patient lives in or has recently travelled to a dengue-endemic area and has symptoms such as fever, vomiting or aches and pains. Warning signs of severe disease include abdominal pain, mucosal bleeding and clinical fluid accumulation, whereas hallmarks of severe disease include severe plasma leakage leading to DSS, severe bleeding and severe endorgan involvement.

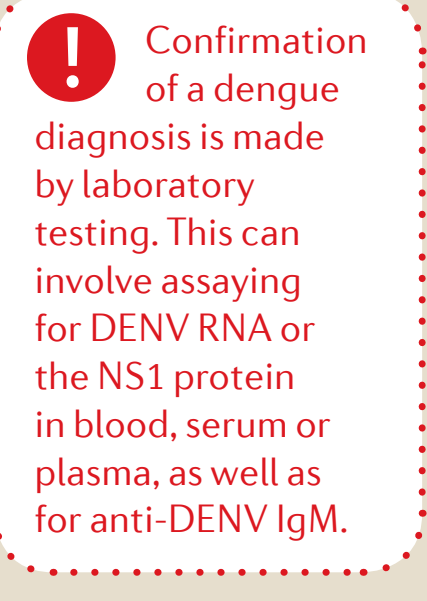

MANAGEMENT

As there is no antiviral therapy for dengue, management involves supportive care. The majority of patients with dengue have mild febrile illness and treatment is commonly bed rest, oral fluids and analgesics. Treatment of dengue with warning signs and severe dengue involves the replacement of fluids lost owing to capillary leakage with

intravenous fluid therapy. Haemorrhages most commonly occur in the gastrointestinal

system, where they can manifest as haematemesis (vomiting of blood)

and/or melaena (black,

tar-like faeces), and are treated

using blood transfusions.

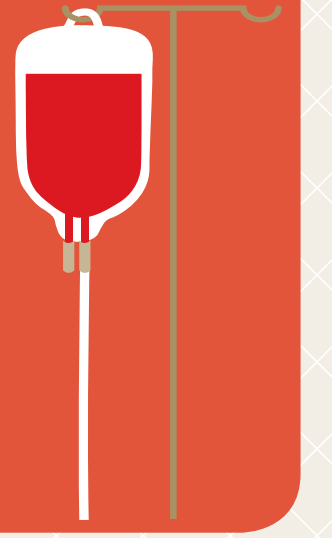

